\title{
La pénurie de personnel enseignant du primaire en Afrique subsaharienne francophone : quels progrès depuis les indépendances?
}

\section{The shortage of primary school teachers in French-speaking sub-Saharan Africa: what progress has been made since independence?}

\section{La escasez de maestros de primaria en el África subsahariana francófona : ¿ ha progresado después de la independencia ?}

\section{Geneviève Sirois, Martial Dembélé et Olivier Labé}

Volume 45, numéro 3, hiver 2018

La quête de l'éducation de qualité pour tous et la profession enseignante dans les pays francophones d'Afrique subsaharienne

URI : https://id.erudit.org/iderudit/1046415ar

DOI : https://doi.org/10.7202/1046415ar

Aller au sommaire du numéro

\section{Éditeur(s)}

Association canadienne d'éducation de langue française

ISSN

1916-8659 (numérique)

Découvrir la revue

\section{Citer cet article}

Sirois, G., Dembélé, M. \& Labé, O. (2018). La pénurie de personnel enseignant du primaire en Afrique subsaharienne francophone : quels progrès depuis les indépendances? Éducation et francophonie, 45(3), 13-34.

https://doi.org/10.7202/1046415ar
Résumé de l'article

Un demi-siècle après les indépendances, les pénuries de personnel enseignant représentent toujours un obstacle majeur à la scolarisation primaire universelle dans plusieurs pays d'Afrique subsaharienne, et plus particulièrement dans les anciennes colonies françaises, où la situation est jugée critique. Pour comprendre ce qui explique cette situation, nous proposons de remonter dans le temps et d'adopter une perspective comparative. Nous avons cherché à comprendre pourquoi les pénuries de personnel enseignant sont un phénomène persistant dans un échantillon de huit pays anciennement colonisés par la France. Il ressort de nos analyses que la décennie 1980-1990, marquée par la mise en oeuvre des programmes d'ajustement structurel, a connu un important ralentissement des progrès des pays pour assurer une offre suffisante d'enseignantes et enseignants pour scolariser un maximum d'enfants. Quant à la période 2000-2015, associée aux objectifs de l'Éducation pour tous, elle a été marquée par une diminution des pénuries d'enseignants. Il apparaît toutefois que les pénuries persisteront dans l'ensemble des pays à l'étude d'ici 2030, notamment en raison des taux de croissance élevés de la population d'âge scolaire et des besoins créés par l'attrition du personnel enseignant.
Tous droits réservés (C) Association canadienne d'éducation de langue française, 2018
Cedocument est protégé par la loi sur le droit d'auteur. L'utilisation des services d'Érudit (y compris la reproduction) est assujettie à sa politique d'utilisation que vous pouvez consulter en ligne. 


\section{La pénurie de personnel enseignant du primaire en Afrique subsaharienne francophone: quels progrès depuis les indépendances?}

Geneviève SIROIS

Université du Québec en Abitibi-Témiscamingue, Québec, Canada

Martial DEMBÉLÉ

Université de Montréal, Québec, Canada

Olivier LABÉ

Institut de statistique de I'UNESCO, Québec, Canada

\section{RÉSUMÉ}

Un demi-siècle après les indépendances, les pénuries de personnel enseignant représentent toujours un obstacle majeur à la scolarisation primaire universelle dans plusieurs pays d'Afrique subsaharienne, et plus particulièrement dans les anciennes colonies françaises, où la situation est jugée critique. Pour comprendre ce qui explique cette situation, nous proposons de remonter dans le temps et d'adopter une perspective comparative. Nous avons cherché à comprendre pourquoi les pénuries de personnel enseignant sont un phénomène persistant dans un échantillon de huit pays anciennement colonisés par la France. Il ressort de nos analyses que la décennie 19801990, marquée par la mise en œuvre des programmes d'ajustement structurel, a 
connu un important ralentissement des progrès des pays pour assurer une offre suffisante d'enseignantes et enseignants pour scolariser un maximum d'enfants. Quant à la période 2000-2015, associée aux objectifs de l'Éducation pour tous, elle a été marquée par une diminution des pénuries d'enseignants. Il apparaît toutefois que les pénuries persisteront dans l'ensemble des pays à l'étude d'ici 2030, notamment en raison des taux de croissance élevés de la population d'âge scolaire et des besoins créés par l'attrition du personnel enseignant.

\section{ABSTRACT}

The shortage of primary school teachers in French-speaking sub-Saharan Africa: what progress has been made since independence?

Geneviève SIROIS, University of Quebec in Abitibi-Témiscamingue, Quebec, Canada Martial DEMBÉLÉ, University of Montreal, Quebec, Canada

Olivier LABÉ, UNESCO Institute of Statistics, Quebec, Canada

Half a century after they became independent, teacher shortages are still a major obstacle to universal primary education in several sub-Saharan African countries, especially in the former French colonies, where the situation is considered critical. To understand this situation, we propose going back in time and adopting a comparative perspective. We tried to understand why teacher shortages are a persistent phenomenon in a sample of eight countries formerly colonized by France. Our analyses show that in the 1980-1990 decade, marked by the implementation of structural adjustment programs, there was a significant slowdown in the countries' progress in terms of ensuring an adequate teacher supply to educate a maximum number of children. Teacher shortages declined in 2000-2015, the period associated with Education for All objectives. However, it appears that shortages will persist in all eight countries until 2030 due to high growth rates of the school-age population and needs created by teacher attrition.

\section{RESUMEN}

\section{La escasez de maestros de primaria en el África subsahariana francófona : ¿ ha progresado después de la independencia ?}

Geneviève SIROIS, Universsidad de Quebec en Abitibi-Témiscamingue, Quebec, Canadá Martial DEMBÉLÉ, Universidad de Montreal, Quebec, Canadá

Olivier LABÉ, Instituto de estadística de la UNESCO, Quebec, Canadá

Medio siglo después de las independencias, la escasez de maestros aun representa un obstáculo mayor en la escolarización primaria universal en varias países de África 
subsahariana, y muy particularmente en las antiguas colonias francesas, en donde la situación se ha juzgado critica. Con el fin de comprender esta situación, proponemos adoptar una perspectiva temporal y comparativa. Hemos tratado de comprender porque la escasez de maestros es un fenómeno persistente en un muestreo en ocho países antiguamente colonizados por Francia. En nuestro análisis aparece que la decena de 1980-1990, marcada por la implementación de programas de ajuste estructural, registró una desaceleración del progreso de los países en el mantenimiento de una oferta suficiente de maestros y maestras para escolarizar al máximo de niños.. En cuanto al periodo 2000-2015, asociado con los objetivos de la Educación para todos, fue marcado por una disminución de la escasez de maestros. No obstante parece que las insuficiencia persistirán en el conjunto de países estudiados de aquí a 2030, principalmente debido a las altas tasas de crecimiento de la población en edad escolar y a las necesidades creadas por la separación del servicio del personal educativo.

\section{PROBLÉMATIQUE}

La décennie 1960-1970 fut marquée par l'accession à l'indépendance de nombreux pays africains. Pour la seule année 1960, 17 pays sur 53 sont devenus indépendants. Au sortir de la colonisation, l'Afrique est le continent le plus en retard en termes de développement de l'éducation. Des recherches (Cogneau, 2003; Labé, Dembélé, Sirois, Motivans et Bruneforth, 2013) ont montré que l'identité du pays colonisateur a eu une influence sur le développement de l'éducation dans les anciennes colonies d'Afrique subsaharienne. En 1960, les taux bruts de scolarisation au primaire et au secondaire étaient plus élevés dans les colonies britanniques $(46,2 \%$ et $6,1 \%)$ que dans les colonies françaises (33,2\% et 1,8\%) (Labé et al., 2013).

Le développement de l'éducation devint donc une priorité. Réunis à Addis-Abeba en 1961 pour discuter du développement des systèmes éducatifs, les États africains formulèrent, pour la première fois, l'objectif de la scolarisation primaire universelle (SPU), dont la réalisation était fixée pour 1980. Les pays devaient cependant faire face aux défis liés au manque d'enseignantes et enseignants qualifiés pour combler les besoins créés par l'expansion de l'offre scolaire et l'africanisation du personnel enseignant, particulièrement dans les anciennes colonies françaises (UNESCOUNECA, 1961). Pour réaliser la SPU à l'horizon 1980, le plan adopté prévoyait le recrutement de 31400 nouveaux enseignants du primaire et de 45300 de plus pour compenser l'attrition (UNESCO-UNECA, 1961). Dès 1960, cependant, l'UNESCO avait souligné que l'un des freins majeurs à la réalisation de cet objectif est la pénurie de personnel enseignant qualifié dans les pays nouvellement indépendants (UNESCO, 1966). 
Un demi-siècle plus tard, les pénuries d'enseignants représentent toujours un obstacle majeur à la réalisation de la scolarisation primaire universelle dans cette région, et plus particulièrement dans les anciennes colonies françaises, où la situation est jugée critique (ISU, 2011, 2016a, 2016b; UNESCO-BREDA, 2009). Qu'est-ce qui explique cette situation? Cet article propose des éléments de réponses à partir d'une analyse rétrospective et comparative. Nous avons cherché à comprendre pourquoi les pénuries d'enseignantes et enseignants sont un phénomène persistant dans un échantillon de sept pays francophones et d'un pays bilingue anciennement colonisés par la France.

\section{CADRE D'ANALYSE: LES PÉNURIES ET LES POLITIQUES DE GESTION DU PERSONNEL ENSEIGNANT}

Les pénuries d'enseignantes et enseignants sont directement liées aux dynamiques de l'offre et de la demande d'enseignants. La région de l'Afrique subsaharienne présente les taux de scolarisation moyens parmi les plus bas du monde, mais également les taux de croissance de la population les plus élevés (ISU, 2006, 2017; UNPD, 2017). La demande d'éducation est donc forte dans la plupart des pays de cette région, ce qui explique que les besoins d'enseignants y soient également importants (ISU, 2016b). Les pénuries d'enseignantes et enseignants résultent d'un déséquilibre entre l'offre et la demande d'enseignants, en raison d'un nombre insuffisant d'enseignants qualifiés et formés pour répondre à la demande ou d'un mauvais déploiement des enseignants (Haggstrom, Darling-Hammond et Grissmer, 1988; ISU, 2006; OCDE, 2005). Dans plusieurs pays, ce déséquilibre pourrait compromettre la scolarisation primaire universelle.

Les pénuries de personnel enseignant peuvent être mesurées à l'aide de trois indicateurs. Le premier est le rapport élèves-enseignant (REE). Il permet de mesurer le nombre d'enseignantes et enseignants dans le système éducatif par rapport au nombre total d'élèves, tous âges confondus (ISU, 2009). Les normes internationales recommandent un REE moyen d'au plus 40 dans l'enseignement primaire.

Quant à l'indicateur d'intensité des pénuries d'enseignants (IIPE), il correspond au rapport entre le nombre d'enfants en âge d'aller à l'école primaire et le nombre d'enseignantes et enseignants en poste. Il permet de mesurer la proportion de ressources humaines investies par rapport au nombre d'enfants à scolariser. De plus, il renseigne sur l'ampleur de la pénurie d'enseignants dans un pays donné advenant que tous les enfants en âge de fréquenter le primaire soient scolarisés (Nkengne, 2010a, 2010b; Sirois, 2017; UNESCO, 2010). Cet indicateur peut aussi être évalué en référence aux normes internationales, qui recommandent un rapport élèves-enseignant de 40. L'IIPE ne renseigne cependant pas sur certains paramètres qui accentuent la demande de personnel enseignant, comme la scolarisation d'enfants plus âgés que l'âge officiel de scolarisation au primaire et le redoublement. Toutefois, il représente 
un indicateur pertinent pour étudier les pénuries passées, en l'absence de données longitudinales fiables et comparables sur les besoins d'enseignantes et enseignants, principalement avant les années 2000.

Le taux de croissance annuel moyen (TCAM) du nombre d'enseignants est le troisième indicateur. Il renseigne sur le flux de l'effectif enseignant (ex.: recrutement, attrition) d'une année à l'autre. L'écart entre le TCAM observé sur une période donnée et celui projeté sur une autre période permet d'évaluer le niveau d'effort attendu par rapport à ce qui a été réalisé dans le passé. Nous utilisons ces trois indicateurs de façon complémentaire dans cet article.

\section{MÉTHODOLOGIE}

\section{Choix des pays}

Le choix des pays à l'étude a été réalisé sur la base de critères relatifs à la date d'accession à l'indépendance, à l'identité du pays colonisateur et aux données disponibles. Des quatorze pays d'Afrique subsaharienne ayant acquis leur indépendance de la France en 1960, nous en avons retenu huit en raison de la disponibilité de données fiables pour la période 1960-2015. L'échantillon comprend sept pays francophones (Bénin, Burkina Faso, République centrafricaine, Congo, Côte d'Ivoire, Mali et Sénégal) et un pays bilingue (Cameroun). Ces pays font partie des plus pauvres de la planète. Il est possible de mesurer les difficiles conditions socio-économiques qui y règnent à partir de l'Indice de développement humain, qui regroupe trois dimensions fondamentales: l'espérance de vie à la naissance, la durée moyenne et la durée attendue de scolarisation ainsi que le revenu national brut par habitant (PNUD, 2016) ${ }^{1}$. Selon le classement des pays en fonction de leur indice de développement humain en 2016, la République centrafricaine figure au $188^{\mathrm{e}}$ rang. Les pays classés dans la catégorie «développement humain faible» incluent la République centrafricaine, le Bénin, le Burkina Faso, la Côte d’Ivoire, le Mali et le Sénégal. Dans la catégorie «développement humain moyen» figurent le Cameroun et le Congo. Comme on peut le voir dans le tableau 1, à l'exception du Cameroun, plus d'une personne pauvre sur deux vit avec moins de $2 \$$ US par jour dans les pays à l'étude. Ceux-ci avaient tous un produit intérieur brut (PIB) par habitant de moins de $250 \$$ US en 1960. En 2015, seuls le Cameroun, le Congo et la Côte d'Ivoire avaient un PIB par habitant supérieur à 1000 \$ US (1374\$ US, 1528 \$S et 1535 \$ US respectivement). En 1960, la Côte d'Ivoire, le Congo et le Sénégal avaient des taux de croissance annuelle de la population de plus de $2 \%$. En 2015, le Mali, le Burkina Faso et le Congo

1. Le Programme des Nations unies pour le développement (PNUD) définit les trois dimensions de l'indice de développement humain (IDH) comme suit: "L'espérance de vie à la naissance exprime la capacité à vivre longtemps et en bonne santé. La durée moyenne de scolarisation et la durée attendue de scolarisation expriment la capacité à acquérir des connaissances. Le revenu national brut par habitant exprime la capacité à avoir un niveau de vie décent. L'IDH a une limite supérieure de 1,0» $(2016$, p. 3). 
affichaient des taux de croissance annuelle de plus de $3 \%$. Enfin, la densité de la population a connu une forte augmentation dans la majorité des pays, particulièrement au Bénin, au Burkina Faso, au Cameroun, en Côte d'Ivoire et au Sénégal. Ces caractéristiques ne sont pas sans effet sur le système éducatif, notamment sur les aspects qui nous intéressent dans cette étude, à savoir l'offre d'éducation ainsi que l'offre et la demande de personnel enseignant.

Tableau 1. Données socio-économiques sur les pays à l'étude

\begin{tabular}{|c|c|c|c|c|c|c|c|c|c|c|}
\hline & & 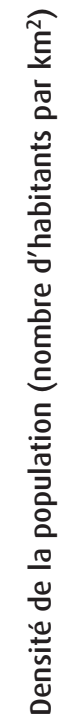 & 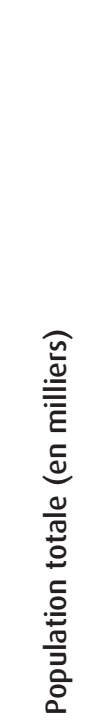 & 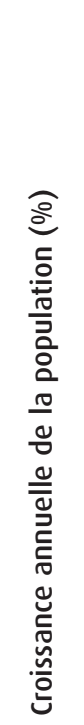 & 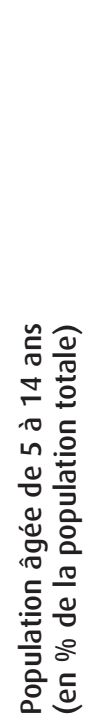 & 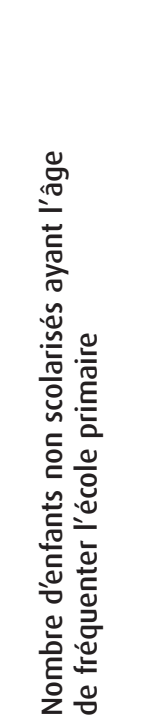 & 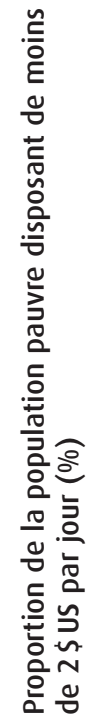 & 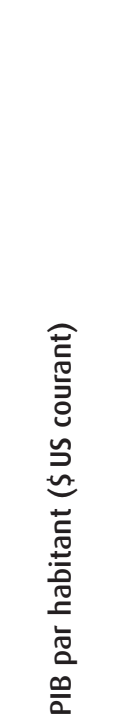 & 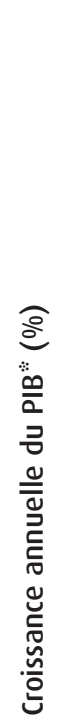 & 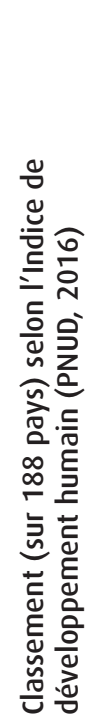 \\
\hline \multirow{2}{*}{ Bénin } & 1960 & 21,6 & 2432 & 1,08 & 22,8 & nd & nd & 93,0 & 3,1 & nd \\
\hline & 2015 & 93,8 & 10576 & 2,84 & 26,6 & 70228 & 75,6 & 789,4 & 4,0 & 167 \\
\hline \multirow{2}{*}{ Burkina Faso } & 1960 & 17,7 & 4829 & 1,34 & 24,6 & nd & nd & 68,4 & 4,0 & nd \\
\hline & 2015 & 66,2 & 18111 & 3,01 & 28,1 & 933180 & 74,6 & 627,1 & 5,9 & 185 \\
\hline \multirow{2}{*}{ Cameroun } & 1960 & 11,0 & 5176 & 1,91 & 23,8 & nd & nd & 119,5 & 1,2 & nd \\
\hline & 2015 & 48,3 & 22835 & 2,73 & 26,7 & 182442 & 43,5 & 1374,5 & 4,5 & 153 \\
\hline \multirow{2}{*}{$\begin{array}{l}\text { République } \\
\text { centrafricaine }\end{array}$} & 1960 & 2,4 & 1504 & 1,43 & 22,3 & nd & nd & 74,6 & 5,0 & nd \\
\hline & 2015 & 7,3 & 4546 & 1,50 & 27,7 & 206651 & 82,3 & 382,2 & 4,5 & 188 \\
\hline \multirow{2}{*}{ Congo } & 1960 & 3,0 & 1037 & 2,39 & 24,4 & nd & nd & 127,0 & 8,4 & nd \\
\hline & 2015 & 14,6 & 4996 & 3,31 & 26,1 & 47349 & 59,6 & 1528,2 & $-1,9$ & 135 \\
\hline \multirow{2}{*}{ Côte d'Ivoire } & 1960 & 11,2 & 3559 & 3,22 & 24,1 & nd & nd & 153,5 & 9,9 & nd \\
\hline & 2015 & 72,7 & 23108 & 2,13 & 26,4 & 746776 & 55,1 & 1535,0 & 8,3 & 171 \\
\hline \multirow{2}{*}{ Mali } & 1960 & 4,3 & 5264 & 1,13 & 23,9 & nd & nd & nd & nd & nd \\
\hline & 2015 & 14,3 & 17468 & 3,27 & 29,2 & 1154062 & 77,7 & 779,9 & 5,8 & 175 \\
\hline \multirow{2}{*}{ Sénégal } & 1960 & 16,7 & 3207 & 2,65 & 25,2 & nd & nd & 242,2 & 3,0 & nd \\
\hline & 2015 & 77,8 & 14977 & 2,76 & 26,4 & 649942 & 66,3 & 958,8 & 6,7 & 162 \\
\hline & & & $\begin{array}{l}\text { le cas de } \\
: \text { Banque }\end{array}$ & & $\begin{array}{l}\text {, il s'agit } \\
017) \text {, ISU }\end{array}$ & $\begin{array}{l}\text { données de } \\
\text { 7) et DPNU }\end{array}$ & $\begin{array}{l}11 \text { et de } \\
17) \text {. }\end{array}$ & & & \\
\hline
\end{tabular}




\section{Sources des données et analyses}

Les données utilisées pour les analyses dont nous présentons les résultats dans la section suivante proviennent de plusieurs sources: les bases de données de l'Institut de statistique de l'UNESCO, l'Annuaire statistique de l'UNESCO (1970), un rapport de la Banque mondiale (1988) et des données de la Division de la population des Nations unies (2017). Les données utilisées par ces organismes s'appuient généralement sur les informations fournies par les pays. Elles sont considérées comme fiables et malgré les potentiels problèmes de comparabilité entre des sources différentes, l'utilisation de plusieurs sources fut le seul moyen d'accéder à toutes les données dont nous avions besoin, notamment pour la décennie 1960-1970.

Nous avons procédé à l'analyse des données en deux temps: une analyse du développement de la scolarisation, suivie d'une analyse de l'évolution des pénuries dans les huit pays à l'étude. Cette dernière comprend une analyse de l'ensemble des pays comme entité et une comparaison entre pays. Pour cette comparaison, nous avons réparti les huit pays selon l'indicateur d'intensité des pénuries d'enseignants (IIPE) en 1960 en nous inspirant de la classification de Labé et al. (2013). Ces auteurs proposent de classer les pays selon le niveau de développement de l'enseignement primaire. Pour notre part, nous avons regroupé les pays en fonction de l'IPPE en 1960. Il en est ressorti trois groupes: le premier est composé des pays ayant un IIPE de plus de 200, soit le Burkina Faso et le Mali; le deuxième groupe comprend les pays dont l'IIPE est situé entre 100 et 200, à savoir le Bénin, la République centrafricaine et le Sénégal; les pays du troisième groupe, le Cameroun, la Côte d'Ivoire et le Congo, ont un IIPE qui se situe entre 50 et 100.

\section{RÉSULTATS}

\section{Développement de l'offre d'éducation primaire dans les huit pays à l'étude}

Le taux brut de scolarisation (TBS) ${ }^{2}$ a servi à analyser le développement de l'offre d'éducation primaire dans les huit pays à l'étude. Comme le montre la figure 1, il y a eu une croissance rapide de la scolarisation dans l'ensemble des pays pendant la période 1960-1980, notamment entre 1960 et 1970 (+18 points de pourcentage), ce qui correspond à la période de la première initiative de scolarisation primaire universelle

2. Nous avons dû faire des choix méthodologiques importants, notamment en ce qui concerne les indicateurs retenus. En effet, les données relatives à des indicateurs plus robustes, tels que le taux net de scolarisation, n'étaient pas disponibles pour la période 1960-1980. Nous avons par conséquent opté pour I'utilisation du taux brut de scolarisation (TBS), un indicateur plus critiqué, mais pour lequel les données sont disponibles pour toute la période de notre étude. Cet indicateur renseigne sur le «nombre d'étudiants scolarisés dans un niveau d'enseignement donné, quel que soit leur âge, exprimé en pourcentage de la population de la tranche d'âges théorique qui correspond à ce niveau d'enseignement » (ISU, 2012a). Le TBS peut parfois dépasser $100 \%$, puisque les élèves plus âgés ou plus jeunes que l'âge officiel pour fréquenter ce niveau d'enseignement ainsi que les redoublants sont inclus. 
en Afrique, lancée à Addis-Abeba en 1961. Cette progression ralentit ensuite, le TBS connaissant une stagnation dans la décennie 1980-1990 et restant sous la barre des $75 \%$ jusqu'en 2000. Cette période correspond aux années des programmes d'ajustement structurel de la Banque mondiale et du Fonds monétaire international, qui imposent à de nombreux pays des restrictions budgétaires importantes en éducation.

Figure 1. Évolution du taux brut de scolarisation (TBS), du rapport élèvesenseignant (REE) et de l'indicateur d'intensité des pénuries d'enseignants (IIPE) au primaire dans les huit 8 pays à l'étude, 1960 à 2015*

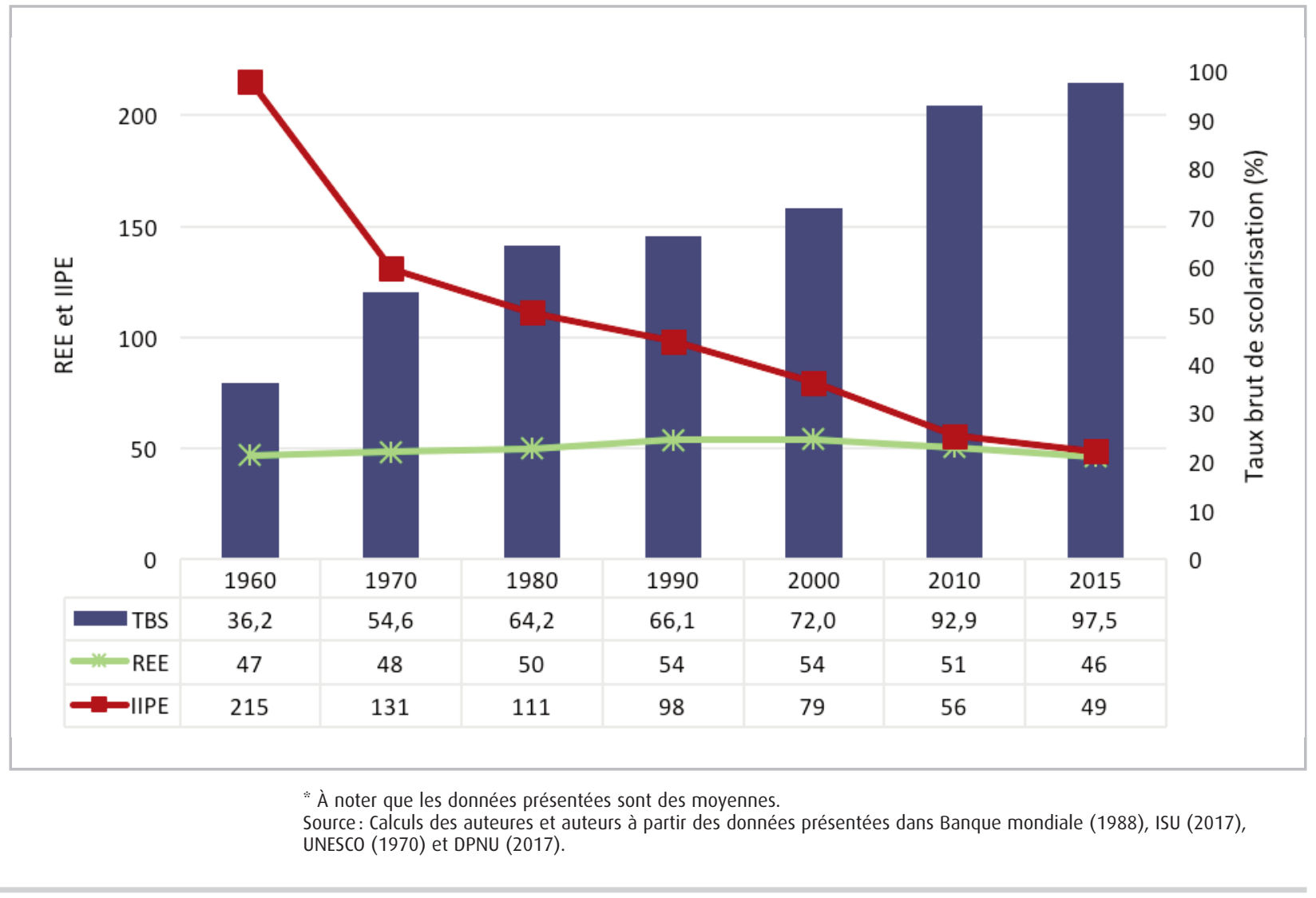

En 2000, en moyenne, un peu plus d'un enfant sur quatre n'a pas accès à l'enseignement primaire. Les progrès les plus importants ont lieu après 2000: le taux brut de scolarisation passe de $72 \%$ à $97,5 \%$ entre 2000 et 2015. Cette augmentation correspond à la période de mise en œuvre des objectifs de l'Éducation pour tous promus 
par l'UNESCO et des objectifs du Millénaire pour le développement des Nations Unies $^{3}$.

Ces constats sont cohérents avec les principales étapes du développement de l'éducation en Afrique subsaharienne décrites par Labé et al. (2013). À partir de l'étude de 22 pays, y compris ceux de notre échantillon à l'exception du Cameroun, ces auteurs distinguent trois étapes: la période 1960-1980, caractérisée par une forte augmentation de l'accès à l'éducation pour tous les ordres d'enseignement (primaire, secondaire, supérieur); la période de 1980 au début des années 1990, caractérisée par une stagnation du développement de l'éducation en raison des restrictions budgétaires; la période allant du début des années 1990 à aujourd'hui, marquée par le développement fulgurant de l'éducation grâce à différentes initiatives internationales (Labé $e t$ al., 2013). Comment l'offre et la demande d'enseignantes et enseignants ont-elles évolué durant ces périodes? C'est à cette question que nous répondrons dans la soussection qui suit.

\section{Évolution des pénuries de personnel enseignant}

\section{Analyse globale de l'évolution des indicateurs}

Notons, de prime abord, que nos analyses confirment l'existence d'une pénurie sévère d'enseignantes et enseignants au lendemain des indépendances. En effet, la moyenne de l'indicateur d'intensité des pénuries d'enseignants l'ensemble des pays à l'étude montre que pour scolariser tous les enfants en âge d'aller à l'école primaire avec le nombre d'enseignants disponible en 1960, chaque enseignante ou enseignant aurait dû être responsable de 215 enfants en moyenne. Par comparaison, selon nos calculs, la moyenne de l'indicateur d'intensité des pénuries d'enseignants de 12 pays anglophones ${ }^{4}$ d'Afrique subsaharienne était de 101 durant la même période. La pénurie était donc deux fois plus sévère dans les pays colonisés par la France que dans les pays colonisés par la Grande-Bretagne. La pénurie a toutefois beaucoup diminué au cours de la décennie 1960-1970, parallèlement à une légère baisse du rapport élèves-enseignant (REE), ces diminutions résultant d'un recrutement considérable d'enseignantes et enseignants conformément au plan d'Addis-Abeba. Les pénuries d'enseignants ont par la suite diminué plus lentement, mais graduellement, jusqu'à 2015. Cependant, le REE est demeuré au-dessus de la norme internationale de 40 au cours des 55 dernières années (47 lors des indépendances contre 46 en 2015).

3. Les objectifs de l'Éducation pour tous ont été définis à Dakar lors du Forum mondial sur l'éducation de 2000 (UNESCO, 2000). Ils sont accessibles à l'adresse suivante: http://unesdoc.unesco.org/images/ 0012/001211/121147f.pdf. Quant aux objectifs du Millénaire pour le développement, ils ont été adoptés lors de la Conférence générale annuelle de I'ONU en 2000. Ils sont accessibles à l'adresse suivante: http://www.un.org/fr/millenniumgoals/.

4. Il s'agit des pays suivants: le Botswana, la Gambie, le Ghana, le Kenya, le Lesotho, le Malawi, Maurice, le Nigeria, la Sierra Leone, I'Ouganda, la Tanzanie et la Zambie. 
Il ressort de ces constats que les phases de l'évolution des pénuries d'enseignants ne suivent pas toujours celles du développement de l'éducation primaire. L'analyse de l'évolution du taux brut de scolarisation (TBS) suggère deux périodes marquées par une tendance considérable à la hausse (1960-1980 et 1990-2015) intercalée d'une période de stagnation (1980-1990). L'analyse de l'évolution du rapport élèves-enseignant et de l'indicateur d'intensité des pénuries d'enseignants montre qu'à la première période de hausse du TBS correspond une importante diminution de l'intensité de la pénurie et une tendance à la hausse du REE. L'augmentation de la scolarisation durant cette période n'a pas été accompagnée d'un accroissement proportionnel des effectifs enseignants, probablement en raison des capacités limitées de formation et du faible bassin de candidates et candidats à la formation des maîtres (ISU, 2006; UNESCO-BREDA, 2009). Durant la période de stagnation du taux brut de scolarisation, l'indicateur d'intensité de pénurie d'enseignants diminue et le rapport élèves-enseignants continue d'augmenter, ce qui suggère un scénario similaire à la période précédente. L'importante augmentation du taux brut de scolarisation après 2000, dans le contexte des objectifs de l'Éducation pour tous, ne se reflète pas non plus sur l'évolution de l'indicateur d'intensité des pénuries d'enseignants, qui diminue lentement, sans que les pénuries soient complètement absorbées. Le rapport élèves-enseignants est à la baisse, mais reste au-dessus de la norme internationale de 40. Cela peut s'expliquer par la scolarisation d'enfants ayant dépassé ou n'ayant pas encore atteint l'âge officiel de fréquenter le primaire. En effet, le phénomène de scolarisation tardive et précoce contribue à augmenter le TBS, mais n'a pas d'impact sur l'indicateur d'intensité de pénurie d'enseignants puisque cet indicateur se base sur la population d'âge scolaire. En revanche, la tendance à la hausse du rapport élèves-enseignants montre que l'augmentation du recrutement d'enseignantes et enseignants n'est pas suffisante pour absorber le flux des nouveaux enfants scolarisés.

\section{Analyse comparative de l'évolution des indicateurs}

Nous proposons ici une comparaison interpays de l'évolution des pénuries de personnel enseignant. Comme on peut le voir dans la figure 2, l'indicateur d'intensité des pénuries d'enseignants connaît une tendance à la baisse. 
Figure 2. Évolution de l'indicateur d'intensité des pénuries d'enseignants (IIPE) par pays, de 1960 à 2015

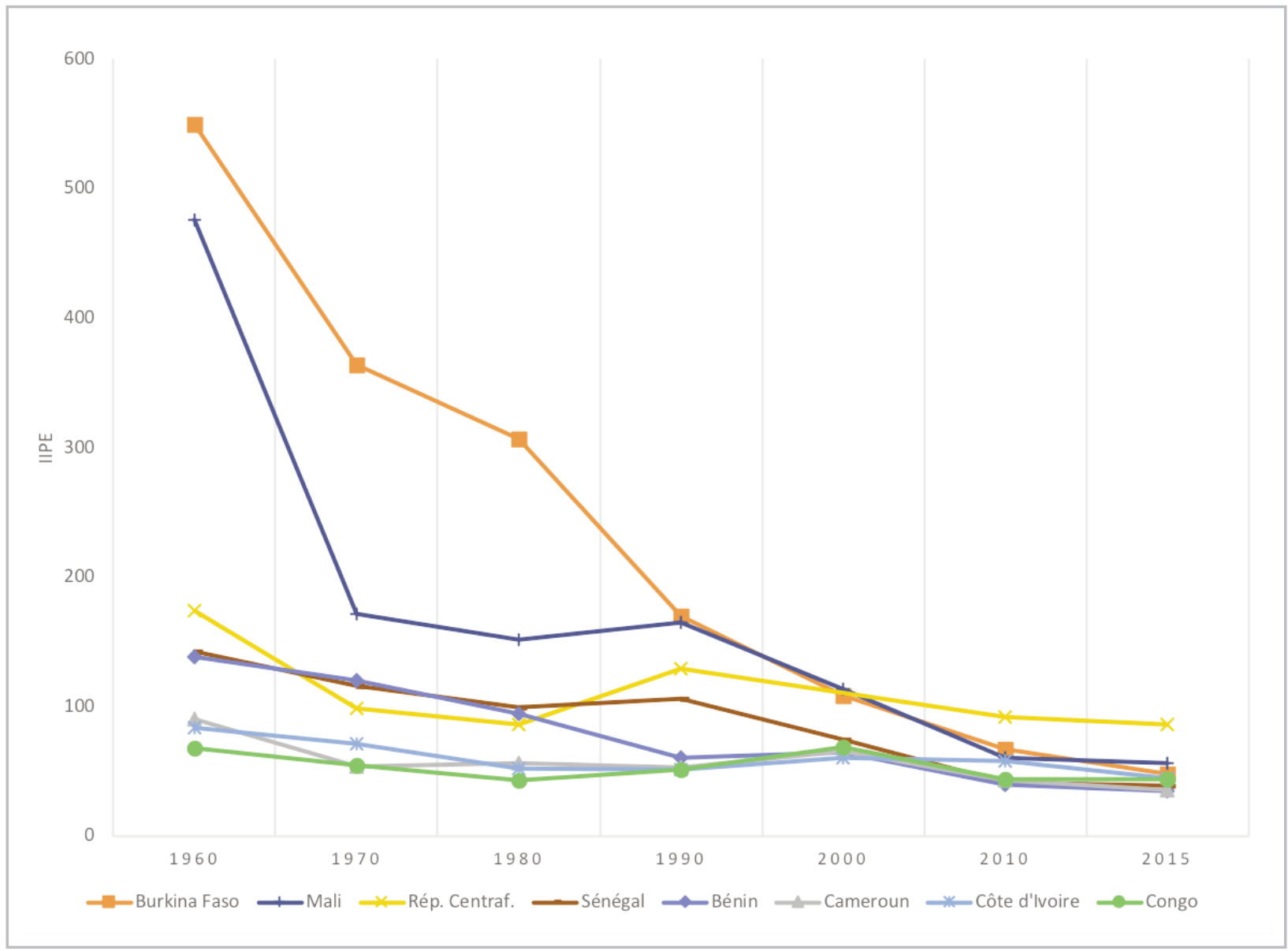

Source: Calculs des auteures et auteurs à partir des données présentées dans Banque mondiale (1988), ISU (2017), UNESCO (1970) et DPUN (2017).

Les trajectoires sont assez semblables pour tous les pays sur la période étudiée, à l'exception du Mali et du Burkina Faso, qui ont des indicateurs d'intensité de pénurie d'enseignants particulièrement élevés lors de l'indépendance, mais qui décroissent substantiellement pour atteindre des valeurs assez voisines de celles des autres pays à partir de 1990. Les disparités du rapport élèves-enseignant entre les pays sont très importantes entre 1980 et 2000 (voir la figure 3), année marquée par une mobilisation internationale en faveur de l'enseignement primaire universel. À partir de 2000, l'évolution de cet indicateur et de celui d'intensité de pénurie d'enseignants dans les différents pays est beaucoup plus homogène avec une tendance à la baisse. La République centrafricaine se démarque par un rapport élèves-enseignants très élevé depuis 1990 et son indicateur d'intensité de pénurie d'enseignants qui est le plus 
élevé de tous les pays à l'étude en 2010 et en 2015. Cela est probablement lié à la crise politico-militaire et humanitaire qui y sévit depuis les années 1990.

Figure 3. Évolution du rapport élèves-enseignant (REE) par pays, de 1960 à 2015

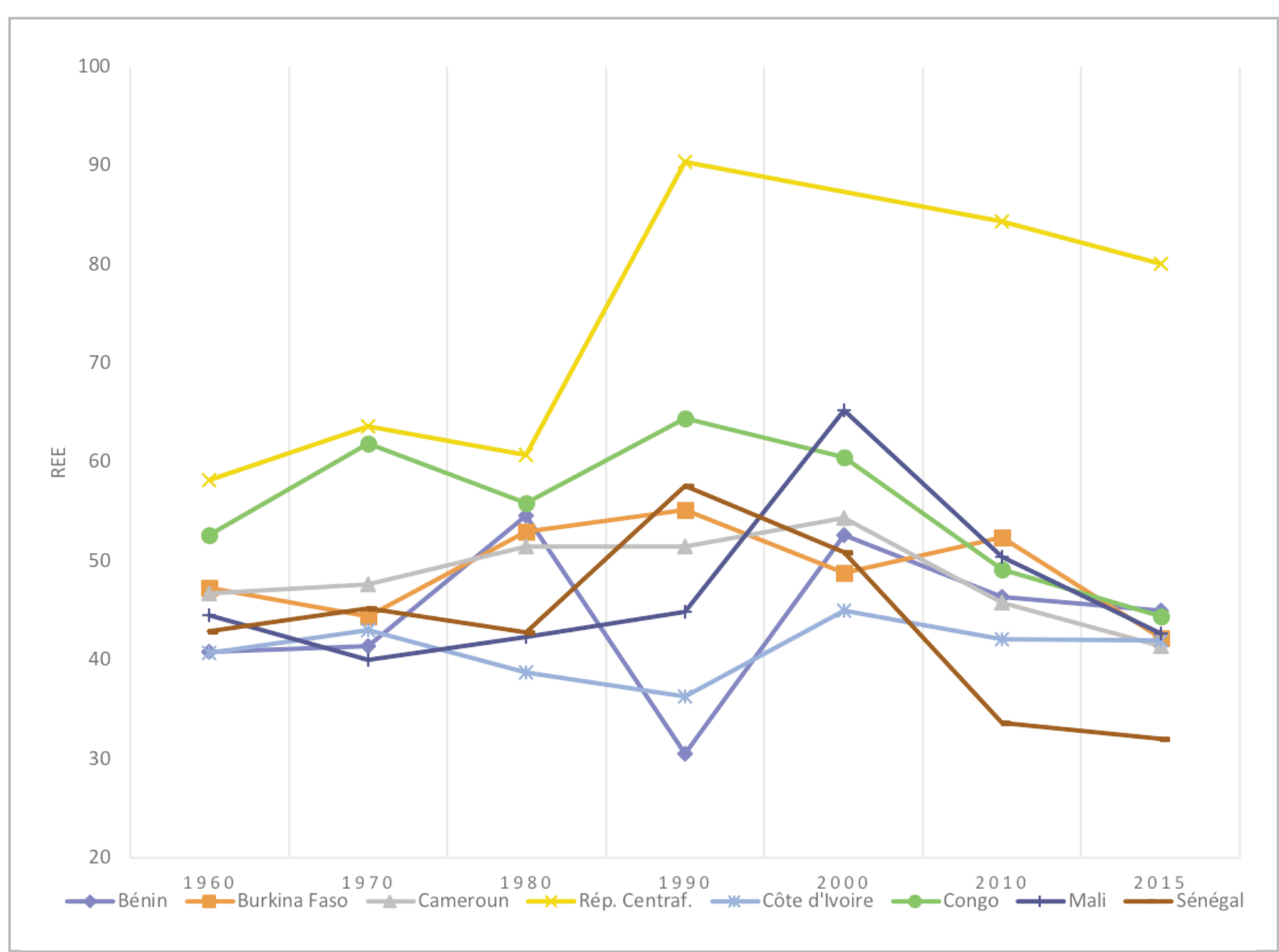

Sources: ISU (2016b) et UNESCO (1970).

Une analyse plus poussée intégrant le taux d'accroissement annuel moyen (TCAM) permet d'examiner les pénuries d'enseignantes et enseignants en parallèle avec le recrutement de nouveaux enseignants. Cette analyse est présentée par groupe de pays, en fonction de l'intensité de la pénurie au moment des indépendances. Le premier groupe est constitué du Burkina Faso et du Mali. Le tableau 2 présente l'évolution des trois indicateurs dans ces deux pays. 
La pénurie de personnel enseignant du primaire en Afrique subsaharienne francophone:

quels progrès depuis les indépendances?

Tableau 2. Évolution des trois indicateurs dans les pays du premier groupe, de $\mathbf{1 9 6 0}$ à 2015*

\begin{tabular}{|c|c|c|c|c|c|c|c|c|}
\hline & & 1960 & 1970 & 1980 & 1990 & 2000 & 2010 & 2015 \\
\hline \multirow{3}{*}{ Burkina Faso } & IIPE & 550 & 364 & 306 & 170 & 109 & 67 & 48 \\
\hline & REE & 47 & 44 & 53 & 55 & 49 & 52 & 42 \\
\hline & TCAM: & nd & $6 \%$ & $4 \%$ & $9 \%$ & $7 \%$ & $8 \%$ & $10 \%$ \\
\hline \multirow{3}{*}{ Mali } & IIPE & 476 & 171 & 152 & 165 & 114 & 61 & 56 \\
\hline & REE & 45 & 40 & 42 & 45 & 65 & 50 & 43 \\
\hline & TCAM & nd & $12 \%$ & $4 \%$ & $2 \%$ & $6 \%$ & $10 \%$ & $5 \%$ \\
\hline \multicolumn{9}{|c|}{$\begin{array}{l}\text { Source: Calculs des auteurs à } \\
\text { DPUN (2017). } \\
\text { "Année la plus proche. } \\
\text { nd: données non disponible. }\end{array}$} \\
\hline
\end{tabular}

Avec respectivement 550 et 476 élèves par enseignante ou enseignant, le Burkina Faso et le Mali présentent les pénuries les plus importantes au lendemain des indépendances, soit deux fois plus que l'indicateur d'intensité de pénurie d'enseignants moyen (IIPE) (215) de l'ensemble des huit pays. Comme l'illustre le tableau 2, ces deux pays ont des trajectoires différentes des autres pays, surtout entre 1960 et 1990. Au Burkina Faso, l'IIPE diminue de manière assez constante, avec un léger ralentissement pendant la période 1970-1980 (TCAM de 4\%) mais une accélération à partir de 1990, pour se situer à 48 en 2015, légèrement sous la moyenne des huit pays (49). Au Mali, le recrutement considérable d'enseignantes et enseignants durant la décennie 1960-1970 (TCAM de 12\%) fait baisser l'indicateur d'intensité de la pénurie d'enseignants (IIPE), qui passe de 475 à 171 (le nombre d'enseignants a été multiplié par 3,5 pendant la période). Il s'agit de la diminution la plus rapide enregistrée parmi les pays à l'étude. Le rapport élèves-enseignant dans les deux pays connaît une succession de baisses et de hausses au cours de la période étudiée. Malgré les efforts déployés pour la scolarisation primaire universelle, ces pays figurent toujours parmi ceux ayant les taux bruts de scolarisation les plus bas, soit $88 \%$ pour le Burkina Faso et $75,8 \%$ pour le Mali. Les pénuries de personnel enseignant y ont diminué mais persistent, puisque les valeurs de l'IIPE restent supérieures à 40 . Cela suggère que les derniers pays ayant obtenu l'indépendance figureraient parmi ceux qui enregistrent, en 2015, d'importants retards quant à la scolarisation des enfants et aux pénuries d'enseignants. La situation est plus critique au Mali, car l'intensité de pénurie d'enseignants se situe largement au-dessus de la norme de 40 et est nettement supérieur à la moyenne de l'ensemble des huit pays. La baisse importante du taux d'accroissement annuel moyen (TCAM) entre 2010 et 2015 (de 5\% à 10\%) coïncide avec les troubles politiques et les problèmes de sécurité majeurs qui éclatent durant cette période, particulièrement dans le nord du pays. En revanche, le Burkina Faso connaît une augmentation du TCAM au cours de la même période, qui passe de $8 \%$ à $10 \%$. 
Parmi les pays du deuxième groupe, caractérisé par des pénuries d'intensité élevée lors de l'indépendance (indicateur d'intensité des pénuries d'enseignants se situant entre 100 et 200), la République centrafricaine se démarque des deux autres et présente une évolution atypique (voir le tableau 3).

\section{Tableau 3. Évolution des trois indicateurs dans les pays du deuxième groupe, de 1960 à 2015*}

\begin{tabular}{|c|c|c|c|c|c|c|c|c|}
\hline & \multirow{3}{*}{$\begin{array}{c}1960 \\
174\end{array}$} & \multirow{3}{*}{$\begin{array}{c}1970 \\
98\end{array}$} & \multirow{3}{*}{$\begin{array}{c}1980 \\
86 \\
\end{array}$} & \multirow{3}{*}{$\begin{array}{c}1990 \\
129 \\
\end{array}$} & \multirow{3}{*}{$\begin{array}{c}2000 \\
\text { nd }\end{array}$} & \multirow{3}{*}{$\begin{array}{c}2010 \\
92\end{array}$} & \multirow{3}{*}{$\begin{array}{c}2015 \\
86\end{array}$} \\
\hline & & & & & & & & \\
\hline \multirow{3}{*}{$\begin{array}{r}\text { République } \\
\text { centrafricaine }\end{array}$} & IIPE & & & & & & & \\
\hline & REE & 58 & 64 & 61 & 90 & nd & 85 & 80 \\
\hline & TCAM & nd & $8 \%$ & $4 \%$ & $-1 \%$ & nd & $4 \%$ & $2 \%$ \\
\hline \multirow{3}{*}{ Bénin } & IIPE & 139 & 120 & 95 & 60 & 65 & 40 & 35 \\
\hline & REE & 41 & 41 & 55 & 31 & 53 & 46 & 45 \\
\hline & TCAM & nd & $5 \%$ & $6 \%$ & $8 \%$ & $3 \%$ & $8 \%$ & $5 \%$ \\
\hline \multirow{3}{*}{ Sénégal } & IIPE & 142 & 116 & 99 & 106 & 75 & 41 & 39 \\
\hline & REE & 43 & 45 & 43 & 58 & 51 & 34 & 32 \\
\hline & TCAM & nd & $6 \%$ & $5 \%$ & $3 \%$ & $6 \%$ & $9 \%$ & $4 \%$ \\
\hline
\end{tabular}

Source: Calculs des auteurs à partir des données présentées dans Banque mondiale (1988), ISU (2017), UNESCO (1970) et DPUN (2017).

"Année la plus proche.

nd: donnée non disponible.

Entre 1960 et 1980, l'indicateur d'intensité des pénuries d'enseignants (IIPE) dans ce pays baisse considérablement avec un taux d'accroissement annuel moyen de $8 \%$ durant la première décennie et de $4 \%$ durant la seconde. Par la suite, l'effectif enseignant du pays diminue, particulièrement pendant la période 1980-1990, en raison du gel des embauches de fonctionnaires (UNESCO, 2007), stimulant à la hausse l'IIPE et le rapport élèves-enseignant (REE). Le nombre d'enseignantes et enseignants en poste a diminué pendant la période, comme le montre la valeur négative du taux d'accroissement annuel moyen. De 1991 à 2005, il n'y a pas de données sur le personnel enseignant. En 2015, les quelques données disponibles indiquent que l'IIPE et le REE de la République centrafricaine sont les plus élevés des huit pays à l'étude. Ces résultats reflètent plusieurs cycles de guerre civile dans le pays depuis 2004 et corroborent dans une certaine mesure le classement actuel du pays selon l'indice de développement humain. La République centrafricaine se place donc à contre-courant de ce qui est observé dans les autres pays. Dans ce contexte, les communautés ont parfois choisi de prendre en charge le recrutement de "maîtres de parents", des enseignants non formés qui sont recrutés et payés localement. Ces enseignantes et enseignants ne sont pas comptabilisés dans les statistiques, puisqu'ils ne font pas partie du système éducatif formel (UNESCO-BREDA, 2009). 
L'évolution des deux autres pays de ce groupe, soit le Bénin et le Sénégal, est assez similaire, à l'exception de la période 1980-2000. Durant cette période, le Sénégal vit de très fortes compressions budgétaires, qui s'accompagnent d'un gel des embauches d'enseignantes et enseignants (UNESCO-BREDA, 2009) et d'une baisse du taux d'accroissement annuel moyen du nombre d'enseignants de deux points de pourcentage (de $5 \%$ à $3 \%$ ) entre 1970-1980 et 1980-1990, alors qu'au Bénin, les effets de l'ajustement structurel se remarquent surtout par une baisse de la fréquentation scolaire (observable par une diminution notable du rapport élèves-enseignant pendant cette période). Par la suite, grâce à une diminution importante de la pénurie d'enseignantes et enseignants, notamment par le biais du recrutement d'enseignants contractuels entre 2000 et 2010 (UNESCO-BREDA, 2009), l'indicateur d'intensité des pénuries d'enseignants de ces deux pays est inférieur à la norme de 40 , ce qui signifie qu'ils disposent théoriquement d'un nombre suffisant d'enseignants pour scolariser tous les enfants en âge de fréquenter le primaire avec un rapport élèvesenseignant de 40. Au Bénin, cependant, le nombre d'enfants scolarisés dépassant ou n'atteignant pas l'âge officiel pour fréquenter le primaire est élevé. Cela engendre des besoins supplémentaires d'enseignantes et enseignants. L'indicateur d'intensité des pénuries d'enseignants (IIPE) est plus faible que le rapport élèves-enseignant (REE), qui est de 45 en 2015. Finalement, les données montrent une diminution assez importante du taux d'accroissement annuel moyen dans les deux pays entre 2010 et 2015 comparativement à la période 2000-2010, suggérant ainsi un ralentissement du recrutement et probablement une compensation de l'attrition avec les nouvelles recrues.

Le dernier groupe comprend le Cameroun, le Congo et la Côte d'Ivoire. Comme le montre le tableau 4, ces pays se caractérisent par des pénuries d'intensité moyenne en 1960 (IIPE inférieur à 100) lors de l'indépendance et évoluent de manière très homogène en ce qui concerne les pénuries de personnel enseignant. 
La pénurie de personnel enseignant du primaire en Afrique subsaharienne francophone:

quels progrès depuis les indépendances?

Tableau 4. Évolution des trois indicateurs dans les pays du troisième groupe, de 1960 à 2015 *

\begin{tabular}{|c|c|c|c|c|c|c|c|c|}
\hline & & 1960 & 1970 & 1980 & 1990 & 2000 & 2010 & 2015 \\
\hline \multirow{3}{*}{ Cameroun } & IIPE & 90 & 53 & 56 & 53 & 65 & 43 & 35 \\
\hline & REE & 47 & 48 & 52 & 51 & 54 & 46 & 41 \\
\hline & TCAM & nd & $6 \%$ & $3 \%$ & $4 \%$ & $1 \%$ & $6 \%$ & $3 \%$ \\
\hline \multirow{3}{*}{ Congo } & IIPE & 67 & 55 & 43 & 51 & 69 & 43 & 44 \\
\hline & REE & 53 & 62 & 56 & 64 & 60 & 49 & 44 \\
\hline & TCAM & nd & $5 \%$ & $6 \%$ & $1 \%$ & $-1 \%$ & $8 \%$ & $4 \%$ \\
\hline \multirow{3}{*}{ Côte d'Ivoire } & IIPE & 84 & 71 & 52 & 51 & 61 & 58 & 45 \\
\hline & REE & 41 & 43 & 38 & 36 & 45 & 42 & 42 \\
\hline & TCAM & nd & $6 \%$ & $9 \%$ & $5 \%$ & $1 \%$ & $3 \%$ & $6 \%$ \\
\hline \multicolumn{9}{|c|}{$\begin{array}{l}\text { Source: Calculs des auteurs à partir de Banque mondiale (1988), ISU (2017), UNESCO (1970) et DPPD (2017). } \\
\text { "Année la plus proche. } \\
\text { nd: donnée non disponible. }\end{array}$} \\
\hline
\end{tabular}

En effet, les pénuries diminuent d'abord rapidement pendant la période 1960-1980 (à l'exception du Cameroun, où cette diminution rapide se limite à la décennie 19601970), puis elles augmentent dans les trois pays jusqu'en 2000. Ils verront le taux d'accroissement annuel moyen du nombre d'enseignantes et enseignants baisser à 1 $\%$ entre 1990 et 2000. L'évolution du taux brut de scolarisation (TBS) montre une baisse importante de la fréquentation scolaire pendant la période 1990-2000 au Cameroun (-14 points) et la période 1980-2000 au Congo (-42 points). Au Cameroun, cette baisse est attribuable à une crise économique qui frappe le pays en 1985 et à la mise en place des programmes d'ajustement structurel (Postlethwaite, 1995). Au Congo, la baisse la plus importante est associée à la période 1990-2000, pendant laquelle le pays est frappé par une guerre civile. En 2015, malgré les difficultés observées, le Congo et le Cameroun figurent parmi les pays du groupe ayant les taux de fréquentation scolaire les plus élevés. En Côte d'Ivoire, deux baisses de la fréquentation scolaire ont été enregistrées au cours de la période d'étude: une entre 1980 et 1990, pendant l'ajustement structurel, et l'autre, plus légère, entre 2000 et 2010. Cette dernière coïncide avec le début de la crise sociopolitique qui a déstabilisé le pays à la suite du coup d'État de 1999 (Pôle de Dakar, 2016).

Dans les trois pays, l'accroissement du recrutement de personnel enseignant après 2000 entraîne une baisse de l'indicateur d'intensité de pénurie qui s'établit, comme le rapport élèves-enseignant, autour de quarante élèves par enseignante ou enseignant en 2015. Ces résultats suggèrent que ces pays disposent actuellement de ressources enseignantes suffisantes pour scolariser tous les enfants en âge de fréquenter le primaire. Cependant, au Congo et au Cameroun, le taux d'accroissement annuel moyen tend à baisser depuis 2010, alors qu'il est en augmentation en Côte d'Ivoire. 


\section{DISCUSSION}

Dès 1960, les pénuries de personnel enseignant ont été considérées par l'Organisation des Nations unies pour l'éducation, la science et la culture (UNESCO) comme l'un des principaux freins au développement de la scolarisation primaire universelle. Nos résultats montrent effectivement que les huit pays francophones étudiés étaient, au moment de leur accession à l'indépendance, en situation de pénurie d'enseignantes et enseignants. Dans cinq de ces pays, pour scolariser tous les enfants en âge de fréquenter le primaire, chaque enseignant aurait dû prendre en charge plus de cent enfants. Le Congo est le pays où la pénurie est la moins sévère en 1960 (IIPE de 67), alors que le Burkina Faso (IIPE de 550) et le Mali (IIPE de 475) ont les déficits les plus importants.

Nos résultats montrent également que les pays où les pénuries étaient les plus importantes lors de l'indépendance (Burkina Faso et Mali) sont aussi ceux où les pénuries d'enseignantes et enseignants ont été les plus persistantes. Ils sont également ceux où les progrès vers la scolarisation primaire universelle ont été les plus lents. Inversement, les deux pays où l'indicateur d'intensité des pénuries d'enseignants (IIPE) diminue le plus rapidement vers la norme de quarante élèves par enseignant sont ceux qui ont les taux bruts de scolarisation les plus élevés actuellement: $117 \%$ en 2015 au Cameroun et $113 \%$ en 2010 au Congo. Dans ces deux pays, l'IIPE est inférieur à 55 à partir de 1970, et une tendance à la baisse se maintient par la suite. En 2015, des pénuries importantes persistent en République centrafricaine (IIPE de 86) et au Mali (IIPE de 56). L'indicateur d'intensité des pénuries d'enseignants (IIPE) des autres pays à l'étude est inférieur à 50 et proche du seuil de 40 élèves par enseignant. Au Cameroun, au Bénin et au Sénégal, l'IIPE se situe en dessous de ce seuil, ce qui suggère que ces pays disposent théoriquement d'un nombre suffisant d'enseignantes et enseignants pour scolariser tous les enfants en âge de fréquenter le primaire tout en s'assurant qu'il y a en moyenne 40 élèves tout au plus par enseignant dans le système éducatif.

Le tableau 5 montre qu'au Bénin et au Sénégal, deux pays où l'indicateur d'intensité de pénurie d'enseignants est actuellement le plus bas de l'échantillon étudié, plus de 60000 nouveaux enseignants doivent être recrutés d'ici 2030 pour combler les besoins liés à l'accroissement de la population d'âge scolaire. Parallèlement, les enjeux associés au remplacement du personnel enseignant qui quitte la profession seront de plus en plus importants dans les années à venir (ISU, 2016b). Selon les projections de l'Institut de statistique de l'UNESCO, les besoins induits par l'attrition dépasseront ceux liés aux nouveaux postes créés pour scolariser tous les enfants dans plusieurs pays. C'est par exemple le cas de la République centrafricaine et du Mali (voir le tableau 5). 
La pénurie de personnel enseignant du primaire en Afrique subsaharienne francophone:

quels progrès depuis les indépendances?

Tableau 5. Estimation du nombre d'enseignantes et enseignants nécessaires pour réaliser la scolarisation primaire universelle dans les huit pays étudiés

\begin{tabular}{|c|c|c|c|c|c|}
\hline & \multirow{2}{*}{$\begin{array}{c}2015 \\
\text { Nombre } \\
\text { d'enseignants } \\
\text { en poste }\end{array}$} & \multicolumn{4}{|c|}{2030} \\
\hline & & $\begin{array}{c}\text { Besoins } \\
\text { combler } \\
\text { l'attrition }\end{array}$ & $\begin{array}{c}\text { Besoins pour } \\
\text { combler les } \\
\text { nouveaux } \\
\text { postes }\end{array}$ & $\begin{array}{c}\text { Nouveau } \\
\text { recrutement } \\
\text { total } \\
\text { nécessaire } \\
\text { d'ici } 2030\end{array}$ & $\begin{array}{c}\text { Taux de } \\
\text { croissance } \\
\text { annuel moyen } \\
\text { requis }\end{array}$ \\
\hline Bénin & 49688 & 12632 & 62548 & 75180 & $6 \%$ \\
\hline Burkina Faso & 64177 & 51119 & 71048 & 122167 & $7 \%$ \\
\hline $\begin{array}{r}\text { République } \\
\text { centrafricaine }\end{array}$ & 8267 & 15965 & 12966 & 28931 & $11 \%$ \\
\hline Cameroun & 105388 & 33818 & 86981 & 120799 & $5 \%$ \\
\hline Congo & 16527 & 10953 & 28672 & 39625 & $8 \%$ \\
\hline Côte d'Ivoire & 80155 & 56647 & 114957 & 171604 & $8 \%$ \\
\hline Mali & 52148 & 68194 & 62882 & 131076 & $9 \%$ \\
\hline Sénégal & 61620 & 51404 & 64996 & 116400 & $7 \%$ \\
\hline
\end{tabular}

Source: Calculs des auteurs à partir des données présentées dans ISU (2016b)

Par ailleurs, le taux de croissance annuel moyen de l'effectif enseignant requis pour scolariser tous les enfants varie entre $5 \%$ et $11 \%$ dans les huit pays étudiés. Or, selon l'ISU (2012b), les pays devant augmenter leurs effectifs d'au moins $3 \%$ chaque année font face à des pénuries aiguës d'enseignantes et enseignants. Les huit pays peuvent donc être considérés, jusqu'en 2030, comme étant en situation de pénurie aiguë. La République centrafricaine apparaît comme l'un des pays où les pénuries seront les plus persistantes au cours des prochaines années. Le nombre d'enseignants doit tripler d'ici 2030 pour répondre aux besoins nationaux. Au Congo, au Mali et en Côte d'Ivoire, leur nombre doit doubler d'ici 2030. La question est de savoir si ces pays pourront soutenir le rythme de recrutement que plusieurs ont connu entre 2000 et 2010. Nous l'avons montré, le taux de croissance annuel moyen du nombre d'enseignantes et enseignants tend à diminuer depuis 2010, ce qui confirme ce que plusieurs experts avaient prévu (UNESCO, 2015, 2017; UNESCO-BREDA, 2009) : les pays semblent présenter des difficultés à soutenir, à long terme, les rythmes de recrutement observés durant cette période, attribuables principalement au recrutement massif d'enseignantes et enseignants contractuels en deçà des normes de qualifications nationales, aux statuts précaires et ayant des salaires souvent dérisoires (Chudgar, Chandra et Razzaque, 2014; Dembélé et Mellouki, 2013; Duthilleul, 2005; Fyfe, 2007; ISU, 2006; Toh, 2017). Vu l'accroissement considérable des besoins liés à l'attrition, il est urgent que les pays trouvent des solutions pour mieux contrôler ce phénomène et retenir les enseignants dans la profession. Un suivi régulier des besoins de personnel enseignant est généralement recommandé parce que les pénuries comblées peuvent réapparaître. En effet, elles dépendent de facteurs dynamiques 
comme la prévalence, parmi les effectifs scolaires, d'enfants plus âgés que l'âge officiel de fréquenter le primaire, le taux de croissance de la population scolarisable et l'attrition des enseignantes et enseignants (ISU, 2014, 2016a, 2016b).

Les pays à l'étude devront en outre prendre des dispositions pour faire face à la pression associée à la demande croissante d'enseignants pour le secondaire, laquelle s'ajoute aux pénuries d'enseignants du primaire.

\section{CONCLUSION}

Dans cette étude, nous avons cherché à comprendre pourquoi les pénuries de personnel enseignant sont un phénomène persistant dans un échantillon de huit pays anciennement colonisés par la France. Grâce à une analyse rétrospective et comparative s'appuyant sur quatre indicateurs pertinents, nous avons fourni des éléments de réponses à cette interrogation. En remontant jusqu'à l'accession de ces pays à l'indépendance, nous avons vu que les phases de l'évolution des pénuries d'enseignantes et enseignants sont les mêmes que celles que nous avons observées pour le développement de l'éducation (Labé et al., 2013). La période 1960-1980, associée à une forte augmentation de l'accès à l'éducation, correspond également à une période de forte intensité du recrutement d'enseignants. La période 1980-1990, caractérisée par une stagnation du développement de l'éducation en raison des restrictions budgétaires liées aux programmes d'ajustement structurel, est marquée dans une majorité de pays par une diminution ou une stagnation du recrutement d'enseignants. Finalement, la période de l'Éducation pour tous (2000-2015), marquée par une scolarisation accrue, enregistre en même temps une baisse considérable des pénuries d'enseignants et des rapports élèves-enseignants avoisinant la norme de 40 dans plusieurs pays, notamment grâce au recrutement massif d'enseignantes et enseignants contractuels.

Les résultats de nos analyses montrent également que le système éducatif est sensible au contexte sociopolitique dans lequel il s'inscrit. Le Congo, la Côte d'Ivoire, le Mali et la République centrafricaine ont particulièrement été aux prises avec diverses crises militaro-politiques depuis plusieurs années. La résolution durable de ces crises se présente comme un défi additionnel qu'ils devront relever pour assurer le développement de leur système éducatif respectif, au service du développement national. 


\section{Références bibliographiques}

Banque mondiale. (1988). Education in Sub-Saharan Africa: Policies for adjustment, revitalization, and expansion. A World bank policy study. Washington, DC: World Bank.

Banque mondiale. (2017). Croissance du PIB (\% annuel) | Data. Repéré à https://donnees.banquemondiale.org/indicateur/NY.GDP.MKTP.KD.ZG

CHUDGAR, A., CHANDRA, M. et RAZZAQUE, A. (2014). Alternative forms of teacher hiring in developing countries and its implications: A review of literature. Teaching and Teacher Education, 37, 150-161. Repéré à http://www.sciencedirect. com/science/article/pii/s0742051X13001571

COGNEAU, D. (2003). Colonisation, school and development in Africa: An empirical analysis. DT DIAL, (1). Repéré à http://www.dial.ird.fr/content/download/49178/ 377873/version/1/file/2003-01.PDF

DEMBÉLÉ, M. et MELLOUKI, M. (2013). The drive towards UPE and the transformation of the teaching profession in French-speaking Sub-Saharan Africa. Dans J. Kirk, M. Dembélé et S. Baxter (dir.), More and betters teachers for quality education for all: Identity and motivation, systems and support (p. 35-53). Sussex, Angleterre: Collaborative Works.

Division de la population des Nations unies (DPNU). (2017). World Population Prospects - Population Division - United Nations. Repéré à https://esa.un.org/ unpd/wpp/DataQuery/

DUTHILLEUL, Y. (2005). Lessons learnt in the use of 'contract' teachers. Paris, France: UNESCO.

FYFE, A. (2007). The use of contract teachers in developing countries: Trends and impact. Working paper. Genève, Suisse: International Labour Organization. Repéré à http://www.ilo.org/wcmsp5/groups/public/---ed_dialogue/---sector/documents / publication/wcms_160813.pdf.

HAGGSTROM, G., DARLING-HAMMOND, L., et Grissmer, D. (1988). Assessing teacher supply and demand. Santa Monica, CA: Rand, Center for the Study of the Teaching Profession.

ISU. (2006). Les enseignants et la qualité de l'éducation: suivi des besoins mondiaux d'ici 2015. Montréal, Québec: UNESCO-ISU.

ISU. (2009). Indicateurs de l'éducation. Directives techniques. Repéré à www.uis.unesco. org/Library/Documents/eiguide09-fr.pdf 
ISU. (2011). La demande mondiale d'enseignants au primaire - Mise à jour 2011. Repéré à http://www.uis.unesco.org/Education/Pages/demande_mondiale_enseignants_ 2011.aspx

ISU. (2012a). Glossaire. Repéré à http://glossary.uis.unesco.org/glossary/fr/home

ISU. (2012b). La demande mondiale d'enseignants au primaire - Mise à jour 2012. Repéré à http://www.uis.unesco.org/Education/Pages/demande_mondiale_enseignants_ 2012.aspx

ISU. (2014). Recherche enseignants formés pour garantir le droit de chaque enfant à l'enseignement primaire. Document de politique 15 / Fiche d'information 30. Repéré à http://www.uis.unesco.org/Education/Documents/fs30-teachers-fr.pdf

ISU. (2016a). Bulletin d'information de l'ISU. Repéré à http://www.uis.unesco.org/ Education/Documents/FS39-teachers-2016-fr.pdf

ISU. (2016b). Teachers projection for primary. Montréal, Québec: ISU.

ISU. (2017). Centre de données. Repéré à http://data.uis.unesco.org/Index.aspx?queryid= 1428 lang $=\mathrm{f} r \#$

Labé, O., Dembélé, M., Sirois, G., Motivans, A. et Bruneforth, M. (2013). Half a century of education progress in Africa (1960-2010). African and Asian Studies, 12, 30-63.

Nkengne, A. P. (2010a). De l'information à la prise de décision, analyse du processus de politique publique en Afrique francophone : le cas de la politique des enseignants contractuels de l'État. Dijon, France: Université de Bourgogne.

Nkengne, A. P. (2010b). Modèle d'explication de l'issue des réformes des politiques publiques en Afrique subsaharienne: illustration avec la politique des enseignants contractuels. Dijon, France: Institut de recherche sur l'éducation.

OCDE. (2005). Le rôle crucial des enseignants. Attirer, former et retenir des enseignants de qualité (éd. Politiques d'éducation et de formation). Paris, France: auteur.

PNUD. (2016). Human Development Report. New York, NY: auteur. Repéré à http:// www.undp.org/content/undp/fr/home/librarypage/hdr/2016-human-developmentreport.html

Pôle de Dakar. (2016). Rapport d'état du système éducatif national de la Côte d'Ivoire. Pour une politique éducative plus inclusive et plus efficace. Repéré à http://unesdoc.unesco.org/images/0024/002470/247040f.pdf

POSTLETHWAITE, T. N. (dir.). (1995). International encyclopedia of national systems of education (2e éd.). Oxford, Angleterre: Tarrytown. 
SIROIS, G. (2017). Attirer, recruter et retenir les enseignants en zones rurales: une analyse des politiques du Burkina Faso en la matière (thèse de doctorat). Université de Montréal, Québec.

TOH, A. (2017). Précarisation de la profession enseignante au primaire en Côte d'Ivoire. Formation et profession, 25(2), 20-34.

UNESCO. (1966). Perspectives africaines. Les progrès de l'éducation. Paris, France: auteur.

UNESCO. (1970). Statistical Yearbook. Paris, France: auteur.

UNESCO. (2000). Cadre d'action de Dakar. L'Éducation pour tous: tenir nos engagements collectifs. Adopté lors du Forum mondial sur l'éducation. Paris, France: auteur.

UNESCO. (2007). Éléments de diagnostic du système éducatif centrafricain (RESEN). Contraintes et marges de manœuvre pour la reconstruction du système éducatif dans la perspective de la réduction de la pauvreté. Repéré à http://unesdoc.unesco. org/images/0015/001514/151488f.pdf

UNESCO. (2010). Guide méthodologique d'analyse de la question enseignante. Dakar, Sénégal: UNESCO-BREDA.

UNESCO. (2015). Rapport mondial de suivi sur l'EPT 2015. Éducation pour tous 20002015: Progrès et enjeux. Paris, France: auteur.

UNESCO. (2017). Accountability in education: meeting our commitments. Repéré à http://unesdoc.unesco.org/images/0025/002593/259338e.pdf

UNESCO-BREDA. (2009). La scolarisation primaire universelle en Afrique: le défi enseignant. Dakar, Sénégal: BREDA.

UNESCO-UNECA. (1961). Final Report. Conference of African states on the development of education in Africa. Addis Ababa, Éthiopie: auteur. 